International Journal of Pure and Applied Mathematics

Volume 105 No. 1 2015, 27-38

ISSN: 1311-8080 (printed version); ISSN: 1314-3395 (on-line version)

url: http://www.ijpam.eu

doi: http://dx.doi.org/10.12732/ijpam.v105i1.4

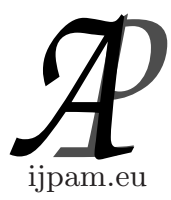

\title{
THE TOPOLOGICAL INDICES OF NON-COMMUTING GRAPH OF A FINITE GROUP
}

\author{
M. Jahandideh ${ }^{1}$, N.H. Sarmin ${ }^{2}$, S.M.S. Omer ${ }^{3}$ \\ ${ }^{1}$ Department of Mathematics \\ Shahid Chamran University of Ahvaz \\ Ahvaz, IRAN \\ ${ }^{2}$ Department of Mathematical Sciences \\ Faculty of Science \\ University Teknologi Malaysia \\ 81310 UTM Johor Bahru, Johor, MALAYSIA \\ ${ }^{3}$ Department of Mathematics \\ Faculty of Science \\ University of Benghazi \\ Benghazi, LIBYA
}

\begin{abstract}
Assume $G$ is a non-abelian finite group. The non-commuting graph $\Gamma_{G}$ of $G$ is defined as a graph with vertex set $G-Z(G)$ in which $Z(G)$ is the center of $G$ and two distinct vertices $x$ and $y$ are joined if and only if $x y \neq y x$. Various topological indices have been determined for simple and connected graphs. Since non-commuting graph is a simple and connected graph, topological indices could be defined for it. The main objective of this article is to calculate various topological indices including the Szeged index, EdgeWiener index, the first Zagreb index and the second Zagreb index for the noncommuting graph of $G$.
\end{abstract}

AMS Subject Classification: $05 \mathrm{C} 12$

Key Words: non-commuting graph, Szeged index, edge-wiener index, the first Zagreb index, the second Zagreb index

Received: June 26, 2015

(C) 2015 Academic Publications, Ltd.

$\S$ Correspondence author url: www.acadpubl.eu 


\section{Introduction}

In this paper, $G$ is a non-abelian finite group. Various graphs could be attributed to $G$, one of which is the non-commuting graph, denoted by $\Gamma_{G}$. The set of vertices and edges of $\Gamma_{G}$ are $V\left(\Gamma_{G}\right)$ and $E\left(\Gamma_{G}\right)$, respectively so that $V\left(\Gamma_{G}\right)=G-Z(G)$ in which $Z(G)$ is the center of $G$ and for every $x, y \in V\left(\Gamma_{G}\right)$ we have $\{x, y\} \in E\left(\Gamma_{G}\right) \Leftrightarrow x y \neq y x$. The centralizer of $x$ within $G$ which is denoted by $C_{G}(x)$ is a subset of $G$ which is defined as $\{g \in G: g x=x g\}$. According to [3], the non-commuting graph of a finite group $G$ was first introduced by Paul Erdos.

Assume that $G=(V, E)$ is a graph in which $V$ is the set of vertices and $E$ is the set of edges. This graph is a finite graph whenever $|V|$ and $|E|$ are finite. The distance between two vertices $x$ and $y$ is denoted by $d(x, y)$, which the length of the shortest path between the two vertices $x$ and $y$. The degree of the vertex $x$ is denoted by $\operatorname{deg}(x)$, equal to the number of edges through $x$. The diameter of $G$ is defined as follows:

$$
\operatorname{diam}(G)=\max \left\{d(x, y): x, y \in V\left(\Gamma_{G}\right)\right\} .
$$

The Szeged index of the graph $G=(V, E)$ is defined as follows: This index is a recently introduced invariant of a graph which is based on the distances of the vertices of the graph [5] and [6]. Let $e=x y$ be an edge of $G$. We define the following sets:

$$
\begin{aligned}
& N_{x}(e \mid G)=\{w \in V: d(w, x)<d(w, y)\}, \\
& N_{y}(e \mid G)=\{w \in V: d(w, y)<d(w, x)\} .
\end{aligned}
$$

Hence $N_{x}(e \mid G)$ is the set of all vertices of $G$ which are closer to $x$ than $y$ and $N_{y}(e \mid G)$ is the set of all vertices of $G$ which are closer to $y$ than $x$. The size of $N_{x}(e \mid G)$ are $N_{y}(e \mid G)$ are denoted by $n_{x}(e \mid G)$ and $n_{y}(e \mid G)$, respectively.

The Szeged index of the graph $G$ is defined by

$$
S z(G)=\sum_{e=x y \in E(G)} n_{x}(e \mid G) \cdot n_{y}(e \mid G) .
$$

Let $G$ be a connected graph. The Edge-Wiener index of $G$ is defined as follows:

$$
W_{e}(G)=\sum_{\{e, f\} \subseteq E(G)} d(e, f) .
$$

Where $e, f$ are two edges in $G$ and $d(e, f)$ is the distance between two vertices in the line-graph. In view of the above definition $W_{e}(G)=W(\bar{G})(\bar{G}$ is the 
line-graph of $G$ ). For more details, refer to the [4]. The first Zagreb index of $G$ is denoted by $Z_{1}(G)$ and is defined by:

$$
Z_{1}(G)=\sum_{x \in V}(\operatorname{deg}(x))^{2} .
$$

The second Zagreb index of the graph $G$ is defined by:

$$
Z_{2}(G)=\sum_{\{x, y\} \subseteq V} \operatorname{deg}(x) \cdot \operatorname{deg}(y) .
$$

The readers can refer to [7] for more details. Our main goal is to calculate the above mentioned indices for the non-commuting graph of $G$ in terms of the order of $G, Z(G)$ and the number of conjugacy classes of $G$. The following lemmas will be used repeatedly.

Lemma 1. [1]. Let $G$ be a finite group. Then $\operatorname{diam}\left(\Gamma_{G}\right)=2$.

Lemma 2. [1]. Let $G$ be a finite group and $k(G)$ the number of conjugacy classes of $G$, then

$$
\left|E\left(\Gamma_{G}\right)\right|=\frac{1}{2}|G|(|G|-k(G)) .
$$

Lemma 3. [1]. Let $G$ be a finite group. If $x$ be one of the vertices of $\Gamma_{G}$, then

$$
\operatorname{deg}(x)=|G|-\left|C_{G}(x)\right|
$$

\section{The Szeged Index of a Non-Commuting Graph}

In this section, we find the Szeged index for the non-commuting graph of a finite group.

Lemma 4. Let $G$ be a finite group. Then

$$
\sum_{x \notin Z(G)}\left|C_{G}(x)\right|=|G|(k(G)-|Z(G)|) .
$$

Proof. We know that $G$ is the union of its conjugacy classes. Assume that $\left\{x_{i}\right\}_{i=1}^{k}$ are the representative of the conjugacy classes and class $\left(x_{i}\right)$ denotes the conjugacy class of $x_{i}$ and $G=\bigcup_{i=1}^{k} \operatorname{class}\left(x_{i}\right)$.

Now, let $\left\{x_{i}\right\}_{i=1}^{t} \notin Z(G)$, thus we have $k(G)=t+|Z(G)|$. Every $x$ which is not 
placed within $Z(G)$ would be placed within one of $\operatorname{class}\left(x_{i}\right) s$ in which $1 \leq i \leq t$. Therefore we have:

$$
\sum_{x \notin Z(G)}\left|C_{G}(x)\right|=\sum_{i=1}^{t}\left|\operatorname{class}\left(x_{i}\right)\right|\left|C_{G}\left(x_{i}\right)\right|=|G| t=|G|(k(G)-|Z(G)|) .
$$

In the next theorem, we calculate the Szeged index of $\Gamma_{G}$.

Theorem 5. Assume $G$ is a finite group and $\Gamma_{G}$ its non-commuting graph. Then the Szeged index of $\Gamma_{G}$ is

$$
\begin{aligned}
S z\left(\Gamma_{G}\right) & =\frac{1}{2}\left(\sum_{i=1}^{n}\left(\sum_{x_{j} \notin C_{G}\left(x_{i}\right)}\left(\left|C_{G}\left(x_{i}\right) \cap C_{G}\left(x_{j}\right)\right|\right)^{2}\right)\right. \\
& +\sum_{i=1}^{n} \operatorname{deg}\left(x_{i}\right)\left(\sum_{x_{j} \notin C_{G}\left(x_{i}\right)}+2\left|C_{G}\left(x_{i}\right) \cap C_{G}\left(x_{j}\right)\right|-\left|C_{G}\left(x_{j}\right)\right|\right) \\
& +|G|\left(\sum_{i=1}^{n}\left(\sum_{x_{j} \notin C_{G}\left(x_{i}\right)}-2\left|C_{G}\left(x_{i}\right) \cap C_{G}\left(x_{j}\right)\right|+\left|C_{G}\left(x_{j}\right)\right|\right)\right) .
\end{aligned}
$$

Proof. Assume that $x$ and $y$ are two arbitrary vertices of the graph $\Gamma_{G}$ that are joined together by $e$ (where $e$ is one of the edges of the non-commuting graph). Now we calculate $n_{x}\left(e \mid \Gamma_{G}\right)$ and $n_{y}\left(e \mid \Gamma_{G}\right)$ :

$$
N_{x}\left(e \mid \Gamma_{G}\right)=\left\{w \in V\left(\Gamma_{G}\right): d(w, x)<d(w, y)\right\} .
$$

According to Lemma 1, we have:

If $d(w, y)=1$ then $d(w, x)=0$ and $w=x$. If $d(w, y)=2$ then $d(w, x)=0$ or 1. So

$$
\begin{aligned}
n_{x}\left(e \mid \Gamma_{G}\right) & =\left(\mid C_{G}(y)-1\right)-\left|C_{G}(x) \cap C_{G}(y)\right|+1 \\
& =\left|C_{G}(y)\right|-\left|C_{G}(x) \cap C_{G}(y)\right|
\end{aligned}
$$

In order to

$$
\begin{aligned}
n_{y}\left(e \mid \Gamma_{G}\right) & =\left(\left|C_{G}(x)\right|-1\right)-\left|C_{G}(x) \cap C_{G}(y)\right|+1 \\
& =\left|C_{G}(x)\right|-\left|C_{G}(x) \cap C_{G}(y)\right| .
\end{aligned}
$$




$$
\begin{aligned}
S z\left(\Gamma_{G}\right) & =\sum_{e=x y \in E} n_{x}\left(e \mid \Gamma_{G}\right) \cdot n_{y}\left(e \mid \Gamma_{G}\right) \\
& =\sum_{e=x y \in E}\left(\left|C_{G}(y)\right|-\left|C_{G}(x) \cap C_{G}(y)\right|\right)\left(\left|C_{G}(x)\right|-\left|C_{G}(x) \cap C_{G}(y)\right|\right) \\
& =\sum_{e=x y \in E}\left|C_{G}(x) \cap C_{G}(y)\right|^{2} \\
& -\sum_{e=x y \in E}\left(\left|C_{G}(x)\right|+\left|C_{G}(y)\right|\right)\left|C_{G}(x) \cap C_{G}(y)\right|+\sum_{e=x y \in E}\left|C_{G}(x)\right|\left|C_{G}(y)\right|
\end{aligned}
$$

Now, we have to calculate the all of summations.

Letting $|G|-|Z(G)|=n$, we obtain $\sum_{e=x y \in E}\left|C_{G}(x) \cap C_{G}(y)\right|^{2}$ :

$$
\sum_{e=x y \in E}\left|C_{G}(x) \cap C_{G}(y)\right|^{2}=\frac{1}{2} \sum_{i=1}^{n}\left(\sum_{x_{j} \notin C_{G}\left(x_{i}\right)}\left(\left|C_{G}\left(x_{i}\right) \cap C_{G}\left(x_{j}\right)\right|\right)^{2}\right)
$$

So we can gain $\sum_{e=x y \in E}\left(\left|C_{G}(x)\right|+\left|C_{G}(y)\right|\right)\left|C_{G}(x) \cap C_{G}(y)\right|$,

$$
\begin{aligned}
& \sum_{e=x y \in E}\left(\left|C_{G}(x)\right|+\left|C_{G}(y)\right|\right)\left|C_{G}(x) \cap C_{G}(y)\right|=\sum_{\substack{x \in G-Z(G) \\
y \notin C_{G}(x)}}\left|C_{G}(x)\right|\left|C_{G}(x) \cap C_{G}(y)\right| \\
= & \sum_{i=1}^{n}\left|C_{G}\left(x_{i}\right)\right|\left(\sum_{x_{j} \notin C_{G}\left(x_{i}\right)}\left|C_{G}\left(x_{i}\right) \cap C_{G}\left(x_{j}\right)\right|\right) \\
= & \sum_{i=1}^{n}\left(|G|-\operatorname{deg}\left(x_{i}\right)\right)\left(\sum_{x_{j} \notin C_{G}\left(x_{i}\right)}\left|C_{G}\left(x_{i}\right) \cap C_{G}\left(x_{j}\right)\right|\right) \\
= & \sum_{i=1}^{n}-\operatorname{deg}\left(x_{i}\right)\left(\sum_{x_{j} \notin C_{G}\left(x_{i}\right)}\left|C_{G}\left(x_{i}\right) \cap C_{G}\left(x_{j}\right)\right|\right) \\
+ & |G| \sum_{i=1}^{n}\left(\sum_{x_{j} \notin C_{G}\left(x_{i}\right)}\left|C_{G}\left(x_{i}\right) \cap C_{G}\left(x_{j}\right)\right|\right.
\end{aligned}
$$

Now, calculating $\sum_{e=x y \in E}\left|C_{G}(x)\right|\left|C_{G}(y)\right|$.

$$
\sum_{e=x y \in E}\left|C_{G}(x)\right|\left|C_{G}(y)\right|=\frac{1}{2} \sum_{i=1}^{n}\left(\left|C_{G}\left(x_{i}\right)\right| \sum_{x_{j} \notin C_{G}\left(x_{i}\right)}\left|C_{G}\left(x_{j}\right)\right|\right)
$$




$$
\begin{aligned}
& =\frac{1}{2} \sum_{i=1}^{n}\left(\left(|G|-\operatorname{deg}\left(x_{i}\right)\right) \sum_{x_{j} \notin C_{G}\left(x_{i}\right)}\left|C_{G}\left(x_{j}\right)\right|\right) \\
& =-\frac{1}{2} \sum_{i=1}^{n}\left(\operatorname{deg}\left(x_{i}\right) \sum_{x_{j} \notin C_{G}\left(x_{i}\right)}\left|C_{G}\left(x_{j}\right)\right|\right) \\
& +\frac{|G|}{2} \sum_{i=1}^{n}\left(\sum_{x_{j} \notin C_{G}\left(x_{i}\right)}\left|C_{G}\left(x_{j}\right)\right|\right) \cdot
\end{aligned}
$$

Now, the Szeged index is equal to

$$
\begin{aligned}
& S z\left(\Gamma_{G}\right)=\sum_{e=x y \in E}\left|C_{G}(x) \cap C_{G}(y)\right|^{2}-\sum_{e=x y \in E}\left(\left|C_{G}(x)\right|+\left|C_{G}(y)\right|\right)\left|C_{G}(x) \cap C_{G}(y)\right| \\
& +\sum_{e=x y \in E}\left|C_{G}(x)\right|\left|C_{G}(y)\right| \\
& =\frac{1}{2} \sum_{i=1}^{n}\left(\sum_{x_{j} \notin C_{G}\left(x_{i}\right)}\left(\left|C_{G}\left(x_{i}\right) \cap C_{G}\left(x_{j}\right)\right|\right)^{2}\right) \\
& +\sum_{i=1}^{n} \operatorname{deg}\left(x_{i}\right)\left(\sum_{x_{j} \notin C_{G}\left(x_{i}\right)}\left|C_{G}\left(x_{i}\right) \cap C_{G}\left(x_{j}\right)\right|\right. \\
& -|G| \sum_{i=1}^{n}\left(\sum_{x_{j} \notin C_{G}\left(x_{i}\right)}\left|C_{G}\left(x_{i}\right) \cap C_{G}\left(x_{j}\right)\right|\right) \\
& -\frac{1}{2} \sum_{i=1}^{n}\left(\operatorname{deg}\left(x_{i}\right) \sum_{x_{j} \notin C_{G}\left(x_{i}\right)}\left|C_{G}\left(x_{j}\right)\right|\right) \\
& +\frac{|G|}{2} \sum_{i=1}^{n}\left(\sum_{x_{j} \notin C_{G}\left(x_{i}\right)}\left|C_{G}\left(x_{j}\right)\right|\right. \\
& =\frac{1}{2}\left(\sum_{i=1}^{n}\left(\sum_{x_{j} \notin C_{G}\left(x_{i}\right)}\left(\left|C_{G}\left(x_{i}\right) \cap C_{G}\left(x_{j}\right)\right|\right)^{2}\right)\right. \\
& +\sum_{i=1}^{n} \operatorname{deg}\left(x_{i}\right)\left(\sum_{x_{j} \notin C_{G}\left(x_{i}\right)}+2\left|C_{G}\left(x_{i}\right) \cap C_{G}\left(x_{j}\right)\right|-\left|C_{G}\left(x_{j}\right)\right|\right)
\end{aligned}
$$




$$
+|G|\left(\sum_{i=1}^{n}\left(\sum_{x_{j} \notin C_{G}\left(x_{i}\right)}-2\left|C_{G}\left(x_{i}\right) \cap C_{G}\left(x_{j}\right)\right|+\left|C_{G}\left(x_{j}\right)\right|\right)\right) .
$$

\section{The Edge-Wiener Index of a Non-Commuting Graph}

In this section, we find the Edge-Wiener index of a non-commuting graph. We start with a couple of lemmas.

Lemma 6. [2]. Assume $G$ is a finite group and $\Gamma_{G}$ its non-commuting graph. If $\overline{\Gamma_{G}}$ is a line-graph then,

$$
\left|V\left(\overline{\Gamma_{G}}\right)\right|=\left|E\left(\Gamma_{G}\right)\right| \quad, \quad\left|E\left(\overline{\Gamma_{G}}\right)\right|=\sum_{x \in V\left(\Gamma_{G}\right)}\left(\begin{array}{c}
\operatorname{deg}(x) \\
2
\end{array}\right) .
$$

Lemma 7. Assume $G$ is a finite group and $\overline{\Gamma_{G}}$ a line-graph of $\Gamma_{G}$. Then $\overline{\Gamma_{G}}$ is a connected graph and $\operatorname{diam}\left(\overline{\Gamma_{G}}\right)=2$.

Proof. First, we prove that there is a path between two vertices of $\overline{\Gamma_{G}}$. Assume that two arbitrary vertices $e$ and $f$ belong to $\overline{\Gamma_{G}}$, thus $e$ is an edge in $\Gamma_{G}$, so there are two vertices $x$ and $y$ of $\Gamma_{G}$ that are joined together by $e$. Furthermore, there are two vertices $x_{1}$ and $y_{1}$ that are connected together by $f$. We know that $\operatorname{diam}\left(\Gamma_{G}\right)=2$, thus there is at least an edge between all mentioned vertices. It means: there is a path between two edges.

Now, we prove that $\operatorname{diam}\left(\overline{\Gamma_{G}}\right)=2$. Suppose that $\operatorname{diam}\left(\overline{\Gamma_{G}}\right)=1$, then $\overline{\Gamma_{G}}$ is a complete graph. Next

$$
\begin{gathered}
\exists x \in G \quad \ni \quad x \neq x^{-1} \Rightarrow \exists y \in G \quad \ni \quad x \stackrel{e}{\rightarrow} y \stackrel{f}{\rightarrow} x^{-1} \\
G \neq C_{G}(x) \cup C_{G}(y) \Rightarrow \exists z \in G-C_{G}(x) \cup C_{G}(y) .
\end{gathered}
$$

Therefore, we have $z \stackrel{h}{\rightarrow} x \stackrel{e}{\rightarrow} y \stackrel{g}{\rightarrow} z$, but $\overline{\Gamma_{G}}$ is a complete graph, so $h$ and $f$ are joined together, which is impossible. Since $z \neq x, y$ and $x \neq y, x^{-1}$. Thus $\operatorname{diam}\left(\overline{\Gamma_{G}}\right) \neq 1$. Hence $\operatorname{diam}\left(\overline{\Gamma_{G}}\right)=2$.

Theorem 8. Let $G$ be a finite group and $\overline{\Gamma_{G}}$ a line-graph of $\Gamma_{G}$. Then

$$
W_{e}\left(\overline{\Gamma_{G}}\right)=\left|E\left(\Gamma_{G}\right)\right|^{2}+|G|^{2}\left(k(G)-\frac{1}{2}|Z(G)|-\frac{1}{2}|G|\right)-\frac{1}{2} \sum_{x \in G-Z(G)}\left|C_{G}(x)\right|^{2} .
$$


Proof. By definition, $W_{e}\left(\overline{\Gamma_{G}}\right)=\sum_{\{e, f\} \subseteq E\left(\Gamma_{G}\right)} d(e, f)=\frac{1}{2} \sum_{e \in E\left(\Gamma_{G}\right)} d(e)$ where

$$
d(e)=\sum_{f \in E\left(\Gamma_{G}\right)} d(e, f) .
$$

First we compute $d(e)$ for an arbitrary vertex of the graph $\overline{\Gamma_{G}}$. According to Lemma $7, d(e)=\sum_{f \in E\left(\Gamma_{G}\right)} d(e, f)=2$ (the number of vertices whose distance from $e$ is 2$)+1$ (the number of vertices whose distance from $e$ is 1 ). Let $x$ and $y$ be joined together by $e$. Then

$$
\begin{aligned}
d(e) & =\sum_{f \in E\left(\Gamma_{G}\right)} d(e, f) \\
& =1((\operatorname{deg}(x)-1)+(\operatorname{deg}(y)-1))+2\left(\left|E\left(\Gamma_{G}\right)\right|-\operatorname{deg}(x)-\operatorname{deg}(y)+1\right) \\
& =2\left|E\left(\Gamma_{G}\right)\right|-(\operatorname{deg}(x)+\operatorname{deg}(y)) .
\end{aligned}
$$

Using the above formula, we can calculate $W_{e}\left(\overline{\Gamma_{G}}\right)$ :

$$
\begin{aligned}
& W_{e}\left(\overline{\Gamma_{G}}\right)=\frac{1}{2} \sum_{e \in E\left(\Gamma_{G}\right)} d(e) \\
& =\frac{1}{2} \sum_{e \in E\left(\Gamma_{G}\right)} 2\left|E\left(\Gamma_{G}\right)\right|-(\operatorname{deg}(x)+\operatorname{deg}(y)) \\
& =\left|E\left(\Gamma_{G}\right)\right|^{2}-\frac{1}{2} \sum_{e \in E\left(\Gamma_{G}\right)}(\operatorname{deg}(x)+\operatorname{deg}(y)) \\
& =\left|E\left(\Gamma_{G}\right)\right|^{2}-\frac{1}{2} \sum_{x \in G-Z(G)}(\operatorname{deg}(x))^{2} \\
& =\left|E\left(\Gamma_{G}\right)\right|^{2}-\frac{1}{2} \sum_{x \in G-Z(G)}\left(|G|-\left|C_{G}(x)\right|\right)^{2} \\
& =\left|E\left(\Gamma_{G}\right)\right|^{2}-\frac{1}{2}|G|^{2}(|G|-|Z(G)|)+|G| \sum_{x \in G-Z(G)}\left|C_{G}(x)\right| \\
& -\frac{1}{2} \sum_{x \in G-Z(G)}\left|C_{G}(x)\right|^{2} \\
& =\left|E\left(\Gamma_{G}\right)\right|^{2}-\frac{1}{2}|G|^{2}(|G|-|Z(G)|)+|G|^{2}(k(G)-|Z(G)|) \\
& -\frac{1}{2} \sum_{x \in G-Z(G)}\left|C_{G}(x)\right|^{2}
\end{aligned}
$$




$$
\begin{aligned}
= & \left|E\left(\Gamma_{G}\right)\right|^{2}+|G|^{2}\left(k(G)-\frac{1}{2}|Z(G)|-\frac{1}{2}|G|\right) \\
& -\frac{1}{2} \sum_{x \in G-Z(G)}\left|C_{G}(x)\right|^{2} .
\end{aligned}
$$

\section{The First Zagreb Index of a Non-Commuting Graph}

In this section, the first Zagreb index of a non-commuting graph is computed.

Theorem 9. Let $G$ be a finite group and $\Gamma_{G}$ its non-commuting graph. Then

$$
Z_{1}\left(\Gamma_{G}\right)=|G|^{2}(|G|+|Z(G)|-2 k(G))+\sum_{x \in G-Z(G)}\left|C_{G}(x)\right|^{2} .
$$

Proof. Using the definition of $Z_{1}\left(\Gamma_{G}\right)$, we have

$$
\begin{aligned}
Z_{1}\left(\Gamma_{G}\right) & =\sum_{x \in G-Z(G)} \operatorname{deg}(x)^{2} \\
& =\sum_{x \in G-Z(G)}\left(|G|-\left|C_{G}(x)\right|\right)^{2} \\
& =|G|^{2}(|G|-|Z(G)|)-2|G| \sum_{x \in G-Z(G)}\left|C_{G}(x)\right|+\sum_{x \in G-Z(G)}\left|C_{G}(x)\right|^{2} \\
& =|G|^{2}(|G|+|Z(G)|-2 k(G))+\sum_{x \in G-Z(G)}\left|C_{G}(x)\right|^{2} .
\end{aligned}
$$

\section{The Second Zagreb Index of a Non-Commuting Graph}

In this section, we calculate the second Zagreb index of a non-commuting graph.

Theorem 10. Let $G$ be a finite group and $\Gamma_{G}$ its non-commuting graph. Then

$$
Z_{2}\left(\Gamma_{G}\right)=\frac{1}{2}\left(|G|^{2}(|G|-k(G))^{2}+|G|^{2}(k(G)-|Z(G)|)-\sum_{1 \leq i \leq n}\left|C_{G}\left(x_{i}\right)\right|^{2}\right) .
$$


Proof. Assume that $x$ is an arbitrary vertex of $\Gamma_{G}$ and is fixed. Now be calculated $\sum_{x \neq y \in G-Z(G)} \operatorname{deg}(x) \cdot \operatorname{deg}(y)$ :

$$
\begin{aligned}
\sum_{x \neq y \in G-Z(G)} \operatorname{deg}(x) \cdot \operatorname{deg}(y) & =\sum_{x \neq y \in G-Z(G)}\left(|G|-\left|C_{G}(x)\right|\right)\left(|G|-\left|C_{G}(y)\right|\right) \\
& =\sum_{x \neq y \in G-Z(G)}\left(|G|-\left|C_{G}(x)\right|\right)|G| \\
& -\sum_{x \neq y \in G-Z(G)}\left(|G|-\left|C_{G}(x)\right|\right)\left|C_{G}(y)\right| \\
& =|G|(|G|-|Z(G)|-1)\left(|G|-\left|C_{G}(x)\right|\right) \\
& -\left(|G|-\left|C_{G}(x)\right|\right) \sum_{x \neq y \in G-Z(G)}\left|C_{G}(y)\right| .
\end{aligned}
$$

We know that $\sum_{y \in G-Z(G)}\left|C_{G}(y)\right|=|G|(k(G)-|Z(G)|)$, thus $\sum_{x \neq y \in G-Z(G)}\left|C_{G}(y)\right|$. Can be found as follows:

$\sum_{x \neq y \in G-Z(G)}\left|C_{G}(y)\right|=|G|(k(G)-|Z(G)|)-\left|C_{G}(x)\right|$ where

$\sum_{x \neq y \in G-Z(G)} \operatorname{deg}(x) \cdot \operatorname{deg}(y)=\left(|G|-\left|C_{G}(x)\right|\right)\left(|G|(|G|-k(G))+\left|C_{G}(x)\right|-|G|\right)$.

Next, we calculate the second Zagreb index of the non-commuting graph. Let $G-Z(G)=\left\{x_{1}, x_{2}, \ldots, x_{n}\right\}$. Then

$$
\begin{aligned}
Z_{2}\left(\Gamma_{G}\right)= & \sum_{\{x, y\} \subseteq V} \operatorname{deg}(x) \cdot \operatorname{deg}(y) \\
= & \frac{1}{2}\left(\sum_{x_{1} \neq y \in G-Z(G)} \operatorname{deg}\left(x_{1}\right) \cdot \operatorname{deg}(y)\right. \\
& +\sum_{x_{2} \neq y \in G-Z(G)} \operatorname{deg}\left(x_{2}\right) \cdot \operatorname{deg}(y) \\
& \left.+\ldots+\sum_{x_{n} \neq y \in G-Z(G)} \operatorname{deg}\left(x_{n}\right) \cdot \operatorname{deg}(y)\right) \\
& =\frac{1}{2}\left[\left(|G|-\left|C_{G}\left(x_{1}\right)\right|\right)\left(|G|(|G|-k(G))+\left|C_{G}\left(x_{1}\right)\right|-|G|\right)\right. \\
& +\left(|G|-\left|C_{G}\left(x_{2}\right)\right|\right)\left(|G|(|G|-k(G))+\left|C_{G}\left(x_{2}\right)\right|-|G|\right) \\
& \left.+\ldots+\left(|G|-\left|C_{G}\left(x_{n}\right)\right|\right)\left(|G|(|G|-k(G))+\left|C_{G}\left(x_{n}\right)\right|-|G|\right)\right]
\end{aligned}
$$




$$
\begin{aligned}
& =\frac{1}{2}\left(|G|^{2}(|G|-k(G))(|G|-|Z(G)|)-|G|(|G|-k(G)) \sum_{1 \leq i \leq n}\left|C_{G}\left(x_{i}\right)\right|\right. \\
& +|G| \sum_{1 \leq i \leq n}\left|C_{G}\left(x_{i}\right)\right|-\sum_{1 \leq i \leq n}\left|C_{G}\left(x_{i}\right)\right|^{2} \\
& \left.-|G|^{2}(|G|-|Z(G)|)+|G| \sum_{1 \leq i \leq n}\left|C_{G}\left(x_{i}\right)\right|\right) \\
& =\frac{1}{2}\left(|G|^{2}(|G|-k(G))^{2}+|G|^{2}(k(G)-|Z(G)|)-\sum_{1 \leq i \leq n}\left|C_{G}\left(x_{i}\right)\right|^{2}\right)
\end{aligned}
$$

\section{Acknowledgements}

The first author would like to thank her supervisor Prof. Darafsheh, professor at Shahid Chamran University. The first author would also like to thank University Teknologi Malaysia for their hospitality in the period 16-July to 15-September 2013 while the author had a visiting position at the university.

\section{References}

[1] A. Abdollahi, S. Akbari, H. R. Maimani, Non-commuting graph of a group, Journal of Algebra, 298 (2006), 468-492.

[2] J. Bondy, G. Murty, Graph Theory with Application. $5^{\text {th }}$ edn. Boston New York: North Holand, (1982).

[3] B. H. Neuman, A problem of Paul Erdos on groups, J. Austral. Soc, Ser. A 21 (1976), 467-472.

[4] P. Dakelmann, I. Gutman, S. Mukwembi, H. C. Swart, The Edge-Wiener index of a graph, Discrete Mathematics, 309 (2009), 3452-3457.

[5] A. A. Dobrynin, I. Gutman, Congruence relations for the Szeged index of hexagonal chains. Uni. Beogr. Publ. Elektrotehn. fak. Ser. Mat. 8 (1997), 106-113.

[6] A. A. Dobrynin, I. Gutman, The Szeged index-a success story. Graph Theory Notes, NY 34 (1998), 37-44. 
[7] S. Nikoli ć, A. Mili čevi ć, N. Trinajsti ć, A. Juric, On use of the variable Zagreb vM2 Index in SPR: Boiling Points of Benzenoid Hydrocarbon, Molecules, 9 (2004), 1208-1221. 